\title{
A History of BlockingQueues
}

\author{
Marina Zaharieva-Stojanovski Marieke Huisman Stefan Blom \\ University of Twente, The Netherlands * \\ m.zaharieva/m.huisman/s.blom@utwente.nl
}

\begin{abstract}
This paper describes a way to formally specify the behaviour of concurrent data structures. When specifying concurrent data structures, the main challenge is to make specifications stable, i.e., to ensure that they cannot be invalidated by other threads. To this end, we propose to use history-based specifications: instead of describing method behaviour in terms of the object's state, we specify it in terms of the object's state history. A history is defined as a list of state updates, which at all points can be related to the actual object's state.

We illustrate the approach on the BlockingQueue hierarchy from the java.util.concurrent library. We show how the behaviour of the interface BlockingQueue is specified, leaving a few decisions open to descendant classes. The classes implementing the interface correctly inherit the specifications. As a specification language, we use a combination of JML and permission-based separation logic, including abstract predicates. This results in an abstract, modular and natural way to specify the behaviour of concurrent queues. The specifications can be used to derive high-level properties about queues, for example to show that the order of elements is preserved. Moreover, the approach can be easily adapted to other concurrent data structures.
\end{abstract}

\section{Introduction}

Writing 'good' formal specifications is an essential factor for efficient program verification. Moreover, these specifications, written in the form of contracts of the software components, provide useful documentation with precisely defined requirements. They should be elegant, readable, independent from the code implementation, and should fully express the required code behaviour.

However, specifying concurrent software is not straightforward. Currently, different techniques are being developed to reason about concurrent programs and specific concurrency features [7, 9, 4], but specifying the functional behaviour of such a program is still a challenge. To address this challenge, this paper introduces a history-based approach to develop behavioural specifications for concurrent data structures. We present the idea on a collection of realistic Java classes, using a combination of JML (the Java Modeling Language) [5] and separation logic [6, 8] as a specification language. This results in clear, readable and abstract specifications.

The major difficulty in specifying the behaviour of concurrent code is caused by thread interference. If threads operate on the same object, a method's postcondition can be unstable, i.e., it can be invalidated by other threads. The following code fragment is part of the put ( $E$ e) method from a shared Queue data structure. The method enqueues an element to the end of the list that internally represents the queue:

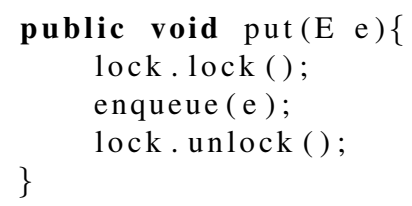

${ }^{*}$ This work was supported by ERC grant 258405 for the VerCors project (all authors), and Artemis grant 2008-100039 for the CHARTER project (Blom).

G. J. Pace and A. P. Ravn:

Proceedings of Sixth Workshop on Formal Languages and Analysis of Contract-Oriented Software (FLACOS'12)

(c) M. Zaharieva-Stojanovski, M. Huisman, S. Blom 
A straightforward way to define the postcondition of this method would be to state that $e$ is the last element in the queue. However, after the lock release another thread may interfere, remove e, and invalidate the postcondition before the caller continues. Therefore, this postcondition would be unstable.

Instead, we propose to maintain a history that records all elements that have been added to the queue. In this way, we can reason about the past. For example, for the put method we can use a history to state that: There was a moment in the past when e was added to the queue.

The main contributions of this work are formal history-based specifications for the classes from the java.util.concurrent.BlockingQueue hierarchy. To make the specifications abstract, modular and naturally organized, we use abstract predicates, specification inheritance and JML model variables. This way we provide an efficient template for easily specifying the classes that implement the BlockingQueue interface, even if they maintain a different order on the elements. Furthermore, we claim that the history pattern is also useful for specifying other concurrent data structures; in particular we have also specified the ConcurrentHashMap using the same idea. Support to reason about historybased specifications will be integrated into the tool set that is currently being developed as part of the VerCors project [1] (Verification of Concurrent Data Structures).

\section{Functional Behaviour Specifications}

In this section we explain how we use histories to overcome the problem of unstable postconditions. Mainly, we discuss the specifications for the BlockingQueue interface, and then we shortly describe how these specifications are inherited by the concrete BlockingQueue instantiation classes.

History-based Specifications A history in our approach is a linked list of nodes that keep the references of all queue elements. The history is defined as a specification ghost variable: /*@ public ghost JMLValueSequence history $=$ new JMLValueSequence ()$; * /$. Therefore, each queue element e has an appropriate history node e' that holds the reference e. We say that e' is a matching node of e. History updates are done by adding JML set statements at the locations where the queue is changed (i.e., an element is added or removed). Since the history should remember all 'old' elements, when a queue element is deleted, its matching history node is not removed, but marked with a negative flag. We define this flag in the node class as a boolean exists variable. The nodes in the history are represented by a model class ListElem $\angle E\rangle$, where $\mathrm{E}$ is the type of the BlockingQueue items:

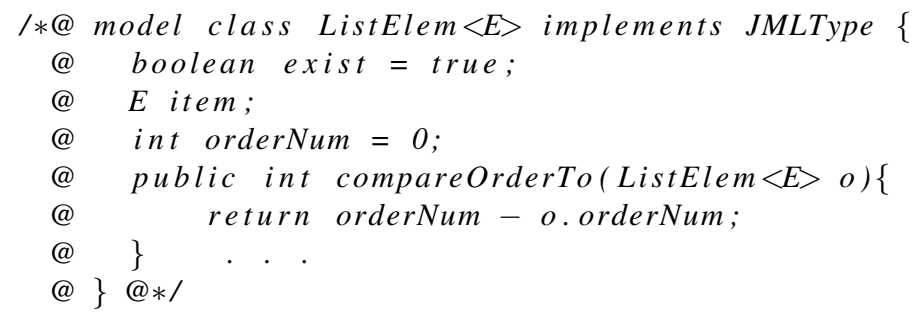

The Order of the Elements Our approach requires preserving the same order of the elements in the history and the queue. Therefore, it is mandatory that each node is added to the correct position in the history list. Nevertheless, different BlockingQueue implementations follow a different order. To keep our approach abstract, we add a compareOrderTo (ListElem $<E>$ ) method in the ListElem class, making the history nodes comparable to each other with respect to their own specific order. We use this method to add a new node to the history at the proper position. 
The common order of the elements in a BlockingQueue is FIFO. Thus, we define a default implementation of the compareOrderTo method with respect to FIFO. Since this order (as well as LIFO) depends on the moment when an element is added to the history, we add the orderNum variable in the ListElem class to represent a counter for the element insertion to the history. Hence, the ListElem is a default history node that respects the FIFO order. The BlockingQueue implementations that follow different ordering of the elements should extend this class and override the compareOrderTo method. An example is the PriorityBlockingQueue where the order of the element depends on the item value.

Methods' Contracts We define the methods' postconditions to express the behaviour of the queue in terms of its history. The postcondition of the add $(E$ e) method states that the history contains the matching node of e. We cannot express anything about the value of its exist flag because it is possible that another thread has removed the element before the end of the method:

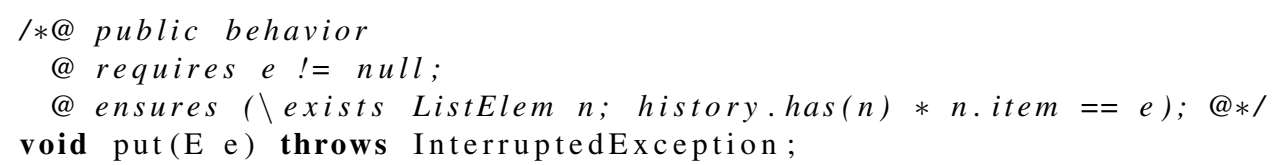

The postcondition of the take() method states that the matching node of the \result (the object returned as a result of this method) exists in the history with a false value of the exist flag. It is not sure that the same node existed before method start, but if it existed, its flag was true.

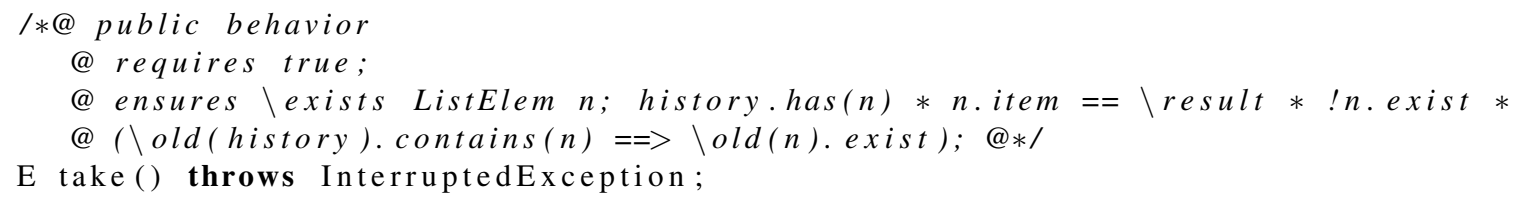

Class Invariant The crucial part of the specifications is to define a property to state that the queue is compatible with the history. This ensures that the queue behaves correctly. Informally, we define a compatibility property stating that: if all nodes with a false flag are removed from the history, the remaining nodes match with the queue elements and are ordered in the same manner (see Fig. 1).

To express this property, we define a recursive predicate compatible, where both parameters (queue and history) are of JMLValueSequence type. We could also have chosen a different type for the queue variable (for example, an array), but we found that this was a simple and elegant way queue to express the predicate.

With the approach presented so far, the order of the history nodes e 1 and $\mathrm{e} 2$ is not precisely defined if e1. compareOrderTo $(e 2)=0$ (in this case we call e1 and e2 equally ordered nodes). Note that equally ordered nodes can exist for example in the PriorityBlockingQueue history. If the nodes A and B in the history (Fig. 1) were equally ordered, we cannot guarantee that A precedes or follows B. Anyhow, the queue and the history should be compatible and therefore, they should follow the same order. The following formal specification of the compatible predicate deals with this case:

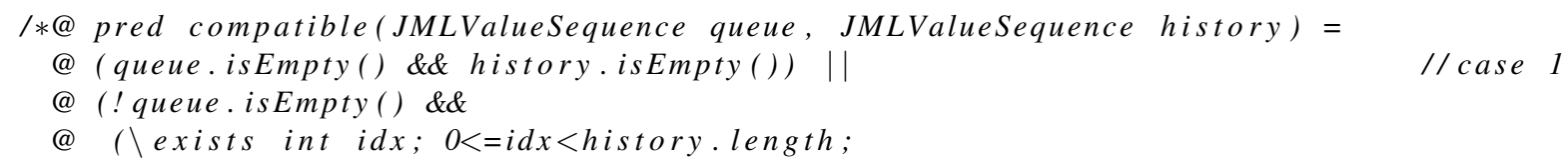




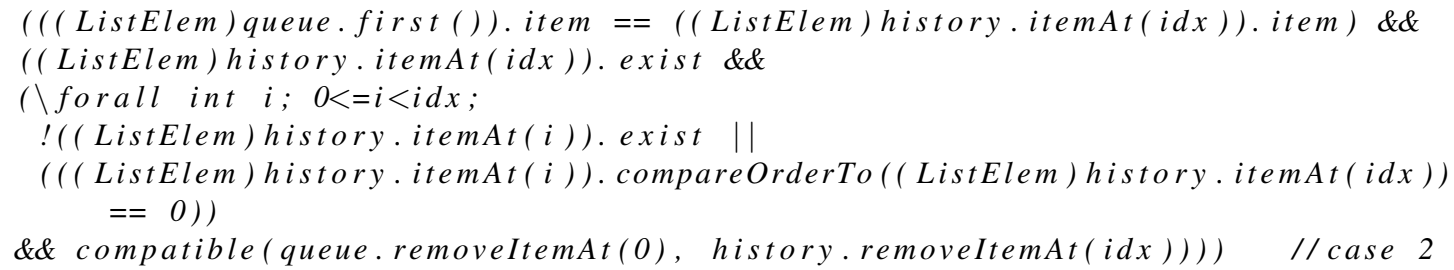

With this definition we even allow expressing the compatibility between queue and a history where the order of elements is not important at all (a set data structure for example). In that case, the method compareOrderTo should be overridden to return always 0 , and the compatible predicate will express that the queue contains the correct elements without considering their order.

Furthermore, a class invariant is specified to state that the queue is compatible with the history. To state the invariant, we use a model variable queue to denote the current contents of the queue.

/*@protected model instance JMLValueSequence queue;

@ invariant compatible(queue, history)@*/

Specifications for the Descendant Classes Specifying the descendant classes is simple: the following steps are required: $(i)$ if the order of the elements in the queue is different then FIFO, extend the ListElem class and override the compareOrderTo (ListElem $<E>$ ) method; (ii) update the history ghost variable at the locations where the queue is changed i.e., an element is added or removed).

The complete specifications together with the Java source code are available via http://www. ewi. utwente.nl/ zaharievam/specs_classes/.

\section{Conclusions}

This paper presents a history-based approach for specifying the functional behaviour of concurrent data structures. It allows one to write stable method specifications, i.e., specifications that cannot be invalidated by other threads. We apply this approach on the java.util.concurrent.BlockingQueue hierarchy, but it is also suitable for specifying other data structures. Furthermore, our approach introduces a new dimension: abstraction. The result is a specification framework, where specifying a new BlockingQueue implementation requires only a small effort.

Related Work Using a history for specifying communication-based parallel programs dates back to the 70 's and 80's. A history, also called a communication trace, is a sequence that records the communication events sent between the processes. The history helps one to reason about the events that happened up to a certain point in time and their order. This approach has been used to specify the interactions in CSP [10]. Also in recent work, a similar idea has been used: Dovland et al. [2] use histories to record the communication events between the components in a distributed environment.

The specifications we provide are closely related to the linearizability-based approach where proving that a data structure is linearizable guarantees its correctness. In this approach the specification contains sequential code that updates a ghost variable, which is an abstract representation of the data structure itself. A library is linearizable with respect to a specification if it behaves as if it was sequentially executed. Linearizability is automatically proved by searching a linearization point for each method [11]. Gotsman et al. [3] explain the importance of linearizability stating that: when proving properties for a client that uses a concurrent library, this library can be soundly replaced by its abstract implementation. 
Our work is more practically oriented. We are focused on making clear and understandable specifications, written in a standard form of method contracts and class invariants. The specifications are readable even without the code, and therefore can be used as formally written documentation.

Future Work The work described here are only the first steps in the VerCors project [1], and there is still much work to be done in the future. A first important step is to develop tool support, so that the history-based specifications can be validated w.r.t. the API reference implementation.

Another point we are currently working on is providing full support for class invariants in a concurrent environment. These class invariants must be strong, i.e., invariants that hold in all possible states. We allow an invariant to explicitly break under certain conditions, while guaranteeing that no other thread is able to observe that it is broken.

It still has to be investigated how the history-based specifications can be exploited to verify client's code. One particular case where this will be useful is if the behaviour of the client depends on the elements that have been in the queue at some point. For example, in a game application, the queue could hold the $k$ highest scores in descending order. A client can use the history-based specifications to derive properties as: the queue holds the best scores, and it knows the correct number of total scores.

Finally, we will also investigate whether we can automatically generate updates to the history, instead of depending on the user manually inserting these updates in the descendant classes.

\section{References}

[1] A. Amighi, S. Blom, M. Huisman \& M. Zaharieva-Stojanovski (2012): The VerCors Project: Setting Up Basecamp. In: Programming Languages meets Program Verification (PLPV 2012), ACM Press, pp. 71-82, doi $10.1145 / 2103776.2103785$

[2] J. Dovland, E. Broch Johnsen \& O. Owe (2008): Observable Behavior of Dynamic Systems: Component Reasoning for Concurrent Objects. ENTCS 203, pp. 19-34, doi:10.1016/j.entcs.2008.04.084

[3] A. Gotsman \& H. Yang (2011): The Importance of Being Linearizable. (Submitted).

[4] C. Haack, M. Huisman \& C. Hurlin (2011): Permission-Based Separation Logic for Multithreaded Java Programs. Nieuwsbrief van de Nederlandse Vereniging voor Theoretische Informatica 15, pp. 13-23.

[5] G.T. Leavens, E. Poll, C. Clifton, Y. Cheon, C. Ruby, D. R. Cok, P. Müller, J. Kiniry \& P. Chalin (2007): JML Reference Manual. Dept. of Computer Science, Iowa State University. Available from http://www . jmlspecs.org.

[6] P. W. O'Hearn, J. Reynolds \& H. Yang (2001): Local Reasoning about Programs that Alter Data Structures. In L. Fribourg, editor: Computer Science Logic, LNCS 2142, Springer-Verlag, pp. 1-19, doi 10.1007/3-54044802-0_1

[7] S. Owicki \& D. Gries (1975): An Axiomatic Proof Technique for Parallel Programs. Acta Informatica Journal 6, pp. 319-340, doi:10.1007/BF00268134.

[8] J.C. Reynolds (2002): Separation Logic: A Logic for Shared Mutable Data Structures. In: Logic in Computer Science, IEEE Computer Society, pp. 55-74, doi:10.1109/LICS.2002.1029817.

[9] G. Schellhorn, B. Tofan, G. Ernst \& W. Reif (2011): Interleaved Programs and Rely-Guarantee Reasoning with ITL. In: Proc. of International Symposium on Temporal Representation and Reasoning in AI (TIME), IEEE Press, doi $10.1109 /$ TIME.2011.12. To appear.

[10] N. Soundararajan (1983): Correctness Proofs of CSP Programs. Theoretical Computer Science 24, pp. 131141, doi $10.1016 / 0304-3975(83) 90045-2$.

[11] Viktor Vafeiadis (2010): Automatically Proving Linearizability. In: CAV, pp. 450-464, doi 10.1007/978-3642-14295-6_40 